\title{
Nordiques
}

39 | 2020

Varia

\section{Le primitivisme emphatique et critique. Les voies du primitivisme finlandais dans Putkinotko de Joel Lehtonen}

\section{Riikka Rossi}

\section{CpenEdition}

Journals

Édition électronique

URL : http://journals.openedition.org/nordiques/704

DOI : $10.4000 /$ nordiques. 704

ISSN : 2777-8479

Éditeur :

Association Norden, Bibliothèque de Caen la mer

Référence électronique

Riikka Rossi, « Le primitivisme emphatique et critique. Les voies du primitivisme finlandais dans Putkinotko de Joel Lehtonen », Nordiques [En ligne], 39 | 2020, mis en ligne le 01 novembre 2020, consulté le 13 mars 2021. URL : http://journals.openedition.org/nordiques/704 ; DOI : https://doi.org/ 10.4000/nordiques.704

Ce document a été généré automatiquement le 13 mars 2021.

Nordiques 


\section{Le primitivisme emphatique et critique. Les voies du primitivisme finlandais dans Putkinotko de Joel Lehtonen}

Riikka Rossi

Introduction

Il est, aux abords de la Carélie, dans le sud du Savo, une contrée belle et sauvage. Le lac Saïmaa, qui, ailleurs, peut être large et dégagé, s'y trouve tellement resserré entre ses rives que rares sont au large les endroits d'où ne soit visible le rivage

opposé, d'où, comme en pleine mer, l'eau ne semble partout se confondre avec l'azur. [...] Les rives sont sauvages et sombres, presque un peu

terrifiantes.

L'intérieur des terres est ici presque aussi sauvage que les bords du lac. Les habitations y sont peu nombreuses, séparées les unes les autres par les mêmes détroits, vallées, golfes et étangs à demi recouverts de mousse. [...] L'aridité des terres et les obstacles créés par les eaux expliquent en partie que, dans cette région,

on puisse trouver des gens qui, en plus d'une pauvreté générale, soient quelquefois si incultes, si indolents, si obstinés, voire si superstitieux et d'un abord si rugueux, qu'ils font presque penser aux sauvages les plus primitifs [...] Et cela est 
d'autant plus étrange que le centre de la civilisation, la ville, ne se trouve qu'à quelques

dix kilomètres. ${ }^{1}$

1 Le roman Putkinotko. Kuvaus laiskasta viinarokarista ja tuhmasta herrasta (1919-1920, tr. en français La Combe aux mauvaises herbes. L'histoire du Bouilleur de cru paresseux et du maitre bête) ${ }^{2}$ de Joel Lehtonen s'ouvre sur une description ambivalente de la forêt finlandaise. Les deux premiers chapitres du roman transportent le lecteur dans un milieu à la fois beau et sauvage, sombre et aride, même un peu terrifiant. La ville, «le centre de la civilisation » se trouve à une distance de dix kilomètres, mais nul chemin n'y mène, sinon de mauvais sentiers grimpant sur les collines. Le peuple de cette région fait penser aux sauvages les plus primitifs, selon le narrateur du roman. Ce dernier, qui observe et documente la région sauvage et ses habitants, représente l'œil civilisé et anthropologique. Étudiant la nature et les paysans des régions isolées de la province du Savo, le roman de Lehtonen dépeint la vie des "hommes de la forêt " et met en parallèle l'existence du peuple finlandais avec celle des indigènes. L'auteur, qui considérait son roman comme un ouvrage sur "la vie d'un peuple sauvage $»,{ }^{3}$ fut inspiré par des études anthropologiques de l'époque portant sur les premières cultures de l'histoire humaine et la vie des peuples exotiques. Lehtonen connaissait bien les travaux d'Edward Westermarck, un anthropologue finlandais, célèbre même sur le plan international. De fait, Lehtonen avait traduit du suédois en finnois une étude de Westermarck, une série de lectures sur sa pensée centrale, Ur sedernas historia (L'histoire des mœurs, 1911)4. Dans l'esprit de Westermarck, qui a étudié les peuples d'Afrique du Nord, Lehtonen s'est également rendu en Afrique et en 1914, il a passé quelques mois en Tunisie. Inspiré par ce voyage, il écrivit un recueil de poèmes intitulé Puolikuun alla. Matkakuvia ja muistelmia murjaanien maasta. (Sous le croissant de lune. Images et mémoires $d u$ pays des Maures) qui parut la même année que la première partie du roman Putkinotko, en $1919^{5}$. Dans ce recueil orientaliste, il compara les nomades du désert aux pauvres gens du monde rural finlandais; les tentes des Bédouins aux taudis sordides des Finlandais.

Dans cet article, nous proposons d'étudier, à travers le roman Putkinotko de Lehtonen, les formes du primitivisme dans la littérature finlandaise et d'examiner les rencontres du primitif et du civilisé dans l'imaginaire de l'époque. Le roman de Lehtonen, en effet, offre un bel exemple du primitivisme dans la littérature finlandaise ${ }^{6}$. La littérature des années 1910 et 1920, que Kai Laitinen appelle «l'âge d'or de la prose finlandaise ", se caractérise surtout comme une grande vague de peinture du peuple finlandais dans le monde rural et primitif7. Dans la littérature de l'époque, les classes agraires sont devenues essentielles pour imaginer le primitif entre nous et pour raconter l'histoire d'une jeune nation à la veille de la modernité8. Au lieu de la ville et de la vie urbaine, l'œuvre de Lehtonen, comme celle de Frans Emil Sillanpää, Ilmari Kianto, Maria Jotuni ou Juhani Aho, met souvent en scène la Finlande des champs et des villages provinciaux. De plus, le monde urbain représenté par ces auteurs est souvent peuplé par un homme primitif, un homme " civilisé », mais troublé par ses passions primitives, ses instincts et les atavismes du passé. Lehtonen fut un cosmopolite, qui séjourna longtemps à l'étranger et connaissait les grandes villes du XIX ${ }^{e}$ siècle par cœur, de Paris à Rome. Néanmoins, dans la sémiologie de Lehtonen, le monde rural et les forêts tenaient un rôle de prédilection ; un monde éloigné et opaque, qui enchante, terrifie et remet en question la modernité et la cité9. Pour Lehtonen, lecteur de Friedrich 
Nietzsche, le côté primitif des forêts finlandaises et de ses habitants représente une source de transgression esthétique et morale, un lieu symbolique pour libérer la passion et l'émotion, contraire au logos et la rationalité supposée de la civilisation.

3 En outre, l'analyse du roman de Lehtonen nous permet de réfléchir sur l'idée du primitivisme en tant que conception esthétique et courant littéraire. Même si le primitivisme a été fréquemment considéré comme un projet colonisant de l'époque impérialiste, il nous semble nécessaire de nuancer et de réviser ce point de vue afin de mettre l'accent sur le potentiel critique du primitivisme littéraire et artistique, voire sur son horizon décolonisant, pour citer Ben Etherington ${ }^{10}$. Comme le souligne Etherington, pour de nombreux auteurs et artistes, le primitivisme marqua une esthétique réactionnaire contre le pouvoir de la politique impérialiste, et au sens large, contre l'ordre social du système capitaliste ${ }^{11}$. En effet, les récits du primitif constituent souvent une rhétorique ambivalente qui défie les hiérarchies de la domination sociale et des attitudes coloniales qu'ils décrivent..$^{12}$ Nous proposons de définir cette conception du primitivisme comme un "primitivisme emphatique ", qui découvre le monde dit primitif du point de vue de l'homme primitif et oppressé, en reconnaissant ses sentiments, et en dirigeant la sympathie du lecteur implicite vers lui ${ }^{13}$. L'effet dégénératif de la civilisation est une idée récurrente dans le primitivisme de l'époque, dans lequel la civilisation occidentale est souvent remise en question. Prenons, par exemple, Heart of Darkness (1902, tr. Au cœur des ténèbres) de Joseph Conrad, célèbre roman sur un voyage le long d'un fleuve au cœur de l'Afrique noire. En décrivant la route vers la jungle ténébreuse, le roman de Conrad dévoile l'exploitation colonialiste et, d'une manière allégorique, suggère qu'en fin de compte le vrai «cœur des ténèbres » se trouve en Occident et non dans le monde primitif.

Ce point de vue critique marque aussi l'œuvre de Lehtonen ${ }^{14}$. Le primitivisme de Putkinotko fait également surgir une transgression vis-à-vis de la civilisation et remet en question le progrès de la modernité. Dans le roman de Lehtonen, la voix du narrateur externe, qui emprunte la rhétorique du discours anthropologique de l'époque, contraste avec le point de vue de l'homme primitif, sa pensée et ses émotions narrées par le discours indirect libre : l'œuvre met en scène son expérience de l'injustice et de l'oppression, ses espoirs et sa nostalgie d'un monde meilleur. En fin de compte, l'homme civilisé se montre sauvage surtout au sens moral, tandis que la famille primitive garde des valeurs morales absentes de la civilisation environnante, comme la solidarité et l'amour du prochain, des valeurs humaines qui sont censées suggérer l'avenir de la nation finlandaise.

5 Néanmoins, il importe ici de souligner que le primitivisme est un concept ambivalent qui a été utilisé pour légitimer divers points de vue idéologiques et politiques. La notion de primitif, en effet, oscille entre deux idées et évaluations presque opposées : ce qui est primordial et pur à l'origine, d'un côté, et ce qui est brut, peu développé et barbare, d'un autre côté15. Le malaise de la civilisation, toutefois, fournit un fil rouge qui nourrit les divers récits du primitivisme. Comme le montrent Arthur Lovejoy et George Boas, dans leur ouvrage classique sur le primitivisme dans l'Antiquité, le désir de sortir de la civilisation peut se manifester sous des formes diverses : la forme chronologique, en tant qu'une nostalgie des premiers temps de l'histoire humaine, ou bien sous la forme culturelle de l'exotisme des cultures isolées de la société occidentale ${ }^{16}$. Il est important aussi de se souvenir qu'il s'agit d'une notion métaphorique qui peut être conçue comme l'autre de la civilisation urbaine. Comme l'indique Marianna Torgovnick dans ses 
études Gone Primitive (1990) et Primitive Passions (1996), d'une certaine manière, le primitif fournit un sous-texte de la modernité, qui n'existe pas sans le primitif ; d'une manière implicite ou explicite, le monde moderne se définit toujours par rapport au primitif qu'il tient à contester ${ }^{17}$. Le rapport entre le primitif et le civilisé est une sorte de chiasme: le moderne n'existe pas sans son autre, et notre idée du primitif est toujours imprégnée par des conceptions de la civilisation occidentale. ${ }^{18}$ Cette hantise de l'archaïque s'étend jusqu'au modernisme littéraire et artistique. Comme le suggère Michael Bell, le modernisme peut être conçu comme une sorte d'anthropologie, qui marqua un retour aux mythes et archétypes pour en constituer une mythopoïétique moderne ${ }^{19}$.

\section{L'homme primitif et ses émotions}

$6 \quad$ Putkinotko de Joel Lehtonen raconte l'histoire d'une famille de métayers pauvres qui nourrissent une haine contre leur propriétaire, et qui, en signe de protestation contre les injustices dans la condition des métayers, laissent sa ferme tomber dans une ruine complète et vivent dans un sauna délabré. Le père de la famille, appelé Juutas Käkriäinen, dont le nom est la version finnoise du biblique "Judas », est comparé à un sauvage : «Oui, son apparence est bien un peu celle d'un animal, que, dans les pays du sud, on appelle un homme des bois ». Avec sa barbiche, ses cheveux et ses poils roux, il possède même des traits démoniaques. Il est ignorant de la société civilisée, mais muni d'un savoir primitif sur la nature. Sa femme Rosina, active et énergique, reste plus ouverte à la société civilisée, mais elle aussi, ressemblant "à une belle indienne », est mise en parallèle avec les sauvages. ${ }^{20}$

7 Conformément à l'idée d'une étude ethnographique, Putkinotko documente la vie quotidienne et les activités ritualisées du peuple rural. Néanmoins, il s'agit d'un roman ambivalent et hybride qui, dans le cadre d'une étude de mœurs réaliste-naturaliste, procède à un mélange des genres. La durée du récit, qui se limite à une seule journée, se conforme à un temps narratif propre à l'écriture moderniste. Le récit est polyphonique: la narration du roman est focalisée sur de nombreux personnages. Écriture amalgame, Putkinotko fusionne la poétique d'idylle avec le grotesque rabelaisien et s'inspire du folklore et de la mythologie finlandaise. La beauté sublime de la nature finlandaise, qui fait surgir la description d'une journée estivale, contraste avec les éléments carnavalesques du récit, les gestes vulgaires et la parole bruyante de la famille de "sauvages ", leurs rires et leur joie de vivre, leurs cris de colère et jurons que fait entendre le discours romanesque. La nostalgie du paradis perdu, le mythe éternel de la littérature et de la culture du début du $\mathrm{XX}^{\mathrm{e}}$ siècle nourrissent l'ambiance à la fois grotesque et mélancolique du roman.

Ce mélange des styles se manifeste aussi dans la peinture des personnages romanesques de Lehtonen qui constituent des types contradictoires. D'une part, la figure d'un métayer paresseux est décrite en opposition au portrait d'un paysan laborieux, l'idéal du peuple pour le mouvement nationaliste du XIXe siècle. Cet archétype du Finlandais fut notamment créé dans la poésie de Johan Ludvig Runeberg (1804-1877) ${ }^{21}$. Le portrait de l'humble peuple finlandais côtoie l'idée du bon sauvage romantique voyant dans le primitif un état « adamique » et « innocent» du premier homme, à la fois un enfant de la nature et un sauvage noble, une figure qui reflète la nostalgie de la nature parmi les classes cultivées. Dans le monde idyllique de Runeberg, le pauvre métayer accepte son 
sort, simplement et sans se lamenter, en pensant que sa vie est entre les mains du Bon Dieu. Les rapports entre les classes supérieures et inférieures entre les pouvoirs autoritaires et le peuple restent harmonieux, étant donné qu'il s'agit de l'ordre naturel, voulu par Dieu. Dans le cadre de ce nationalisme romantique, la pauvreté peut être acceptée, parce qu'il ne s'agit que d'une phase temporaire vers une société meilleure. L'amour de la patrie a souvent été conçu comme une sorte d'attente : le patriotisme se fonde sur la promesse de la réalisation d'un idéal national. L'attente de la réalisation de cette promesse engage les gens à travailler pour la nation, à endurer des moments difficiles, même à se sacrifier pour elle. ${ }^{22}$

9 Le roman Putkinotko de Joel Lehtonen refuse, pourtant, de garder cette harmonie de l'idylle, l'attente de l'égalité à l'avenir et thématise le conflit entre les classes sociales. La condition difficile des métayers fut un des problèmes qui ont conduit à l'éclatement de la guerre civile finlandaise de 1918. La question sur le droit de posséder la terre, qui a été tenue pour une des raisons de la pauvreté rurale en Finlande ${ }^{23}$, contribue au primitivisme du roman: en représentant les pauvres métayers en tant que des indigènes oppressés et privés de la terre natale qu'ils cultivent, Lehtonen interprète la politique agraire de l'époque comme une forme de colonialisme interne. ${ }^{24}$ Le roman met en scène les faiblesses dans le statut d'un locataire de la terre et relève de la réalité historique. Depuis une vingtaine d'années, la famille Käkriäinen a vécu avec la peur d'être expulsée de sa maison. Le propriétaire de Putkinotko menace de les chasser s'ils ne réalisent pas les travaux et les réparations qu'il impose. Même un accord convenable leur a manqué : au début, il n'y eut qu'un accord verbal concernant leur droit d'y habiter. Ensuite, un contrat écrit fut signé. Le nom du propriétaire, Muttinen (mutta $=$ «mais » en finnois) illustre l'hésitation qui le caractérise, et cristallise son incapacité à résoudre le statut de ses métayers: ses promesses d'un avenir meilleur sont toujours accompagnées d'un " mais », indiquant une condition souvent impossible à atteindre par les métayers.

10 De cette manière, le roman de Lehtonen pose la question des inégalités économiques dans le monde rural, et même celle de l'exploitation des pauvres gens, dont la corvée permet aux propriétaires de mener une vie confortable. Dans le primitivisme critique de Lehtonen, les émotions et l'empathie narrative ${ }^{25}$ jouent un rôle important : le lecteur implicite du roman est amené à reconnaître les sentiments des primitifs sous l'oppression et à sentir une indignation morale face aux injustices sociales du monde rural. La perspective des pauvres gens, représentée par le discours indirect libre et la focalisation interne, permet au lecteur de se mettre à la place des personnages, de se reconnaître, et même de s'identifier à eux avec la gamme de leurs émotions : espoirs, joie et déception, mélancolie et honte, et même jusqu'à la haine et la colère suscitées par l'inégalité. Le point de vue de la mère de la famille, Rosina, joue un rôle important. La narration focalisée par la mère révèle son souci de l'avenir, pour ses enfants en particulier. La terre qui nourrit la famille éveille l'amour en elle, mais le sentiment d'injustice suscite une haine profonde :

Mais, en même temps, la jalousie et la rage se mêlent à ces pensées agréables sur le champ, qui a été défriché et travaillé par eux, les habitants de Putkinotko, et qui n'est pas vraiment et complètement à elle ou à eux. Cette amertume lui brûle la gorge comme du sel. ${ }^{26}$

11 À l'instar de son mari, elle en a assez de l'incertitude de l'avenir qui les tourmente. Sa résolution fait penser aux figures de la femme forte dans le naturalisme critique de Minna Canth et de ses confrères. ${ }^{27}$ En outre, il est à noter que Rosina canalise son 
mécontentement vers une colère énergique, tandis que le père de la famille, Juutas, est enclin à sombrer dans une mélancolie passive qui le mène à une incapacité à agir, ou, alternativement, même à des pensées destructives :

Juutas ressent une douleur dans la poitrine. Une terrible douleur. Cela fait plus de dix ans qu'il demeure à Putkinotko, et il a même pensé à défricher le marécage. Maintenant, les autres vont lui prendre tout ce qu'il y a déjà de fait. Et ils vont prendre leurs affaires, à eux. Faudrait tout démolir. Faire place nette à coups de pied. $^{28}$

12 En même temps que Lehtonen fait surgir la douleur du pauvre métayer, le passage cité ci-dessus évoque aussi l'aspect comico-grotesque du roman : l'auteur implicite raille de Juutas, le paresseux, qui a "même pensé à défricher le marécage ». Les lectures de Putkinotko ont mis l'accent sur l'interprétation du père comme un stéréotype d'un fainéant, jusqu'à une caricature du paresseux. ${ }^{29}$ Toutefois, en tenant compte de la tragédie qui réside au fond, c'est un rire sombre. L'inaction du père peut être conçue comme une forme de résistance passive contre la société qui n'offre pas de voie pour sortir de la pauvreté. La description de la dépression du métayer sert à critiquer la société de l'époque; de la même manière que la colère, l'émotion négative, ici, se transforme en une forme de résistance politique.

Du point de vue du lecteur implicite, la description de l'oppression des basses classes est destinée à susciter l'indignation et la colère qui, depuis Aristote, sont considérées comme une émotion morale. La reconnaissance de leur expérience et de leurs émotions peut stimuler le sens éthique du lecteur. Le pouvoir critique des émotions négatives se manifeste aussi au niveau du récit : la colère de la mère de la famille, Rosina, fait peur au propriétaire de la ferme, l'éveille à la reconnaissance de leur misère et lui fait comprendre les défauts dans la condition des métayers :

Quelle haine profonde et passionnée ne voyais-je pas bouillonner dans les yeux de Rosina quand j'ai obligé son mari à accepter mes articles ! Et te rappelles-tu ce que disait Rosina, surtout l'été dernier? Si elle nous remarquait dans la cour de la cabane ou sur la lisière du champ, elle déchargeait son cœur, en apparence à l'adresse des autres Käkriäinen, mais en réalité ses paroles m'étaient destinées. Elle disait: pourquoi est-ce qu'on labourerait et sèmerait ici...dans le domaine d'un autre, qui peut être chassé ! $!^{30}$

Ainsi, le propriétaire de la ferme réfléchit au rôle de la famille des métayers à la fin du roman. En fin du compte, pour le propriétaire, il est facile de se mettre à la place de ses locataires, étant donné qu'il est lui-même un parvenu, d'origine pauvre ; il se souvient toujours de la sensation de faim qui le tenaillait pendant son enfance. Après une longue hésitation, il décide de donner une partie de la terre à la famille. Certes, on peut se demander si cette décision n'arrive pas trop tard, car la famille finit ruinée. À la fin du roman, la belle journée d'été se tourne vers une nuit sombre, et un incident entre les métayers et le propriétaire suggère que la tension entre les classes devient critique. Le fils aîné de la famille, Ananias, qui est ivre, tire un coup de feu accidentel sur le lac, et la maitresse du propriétaire, Lyygia, répond en tirant avec son revolver ; ces coups de feu évoquent l'éclatement de la guerre civile en 1918. Dans le recueil de nouvelles Korpi ja puutarha (La forêt et le jardin), en 1923, Lehtonen continue l'histoire de la famille Käkriäinen. «La fin des sauvages des forêts, c'est toujours tragique ", ${ }^{31}$ écrit-il. Puis, il livre un épilogue sombre : la famille, dont la vitalité et la joie étaient mises en scène dans Putkinotko, est tombée dans une pauvreté encore pire ; ses membres sont séparés 
les uns des autres; le père meurt en prison après avoir été arrêté pour distillation illégale.

\section{Le malaise dans la civilisation, la nostalgie pour la nature authentique}

15 Les passions primitives, de la colère et de l'agression à la joie spontanée, fournissent effectivement un caractère distinctif de la famille Käkriäinen. En ce qui concerne leur spontanéité émotionnelle, la description des personnages de Putkinotko emprunte des idées provenant de l'anthropologie de l'époque, selon lesquelles un homme primitif, prélogique et mystique, est plus enclin à s'abandonner au pouvoir de ses émotions ${ }^{32}$. Néanmoins, dans le roman de Lehtonen, le cliché colonisant d'un homme primitif simple et irrationnel s'est transformé. Le point de vue des personnages romanesques révèle les structures de pouvoir et l'inégalité sociale; leurs émotions font surgir le savoir moral inscrit dans le sentiment d'injustice. L'idéal de la liberté émotionnelle, qui s'inscrit dans la passion des personnages de Lehtonen, a souvent été conçu comme une condition dans le sens du pouvoir et de l'autonomie d'un individu. ${ }^{33}$ L'accent mis sur la libre expression des émotions fournit un contre-pouvoir à la rationalité supposée de la société civilisée et fait penser au "malaise dans la civilisation », aux restrictions de la culture moderne, comme le souligne Sigmund Freud dans son étude célèbre Das Unbehagen in der Cultur (1929, Le malaise dans la civilisation).

Il est important de noter que, même si la colère, la haine et l'indignation, y compris un sentiment proprement finlandais, sisu - la résilience ou la persévérance ${ }^{34}$ - forment les émotions centrales des personnages romanesques, la colère suscitée par les injustices ne s'exprime pourtant pas par la violence ou l'agression au niveau de l'action. ${ }^{35} \mathrm{Au}$ contraire, Lehtonen souligne le caractère pacifique de ses personnages, leur amour du prochain et leur solidarité familiale. Habillés de leurs vêtements sales et en loques, vivant dans un sauna infesté de mouches et de punaises, la famille naturaliste de Lehtonen conteste les idéaux de l'ordre et de la propreté de la civilisation, qui caractérisèrent l'idéal d'un vrai Finlandais dans la pensée de l'époque. Pourtant, au niveau éthique et moral, les personnages de Lehtonen se révèlent être des hommes engagés qui prennent soin les uns des autres et montrent des valeurs morales absentes de la civilisation environnante. Malgré leurs disputes récurrentes, le mariage de Juutas et Rosina reste loin d'une tragédie naturaliste. Tous deux font preuve d'amour et de confiance, l'un à l'égard de l'autre. Leurs enfants peuvent être désobéissants, mais ils leur apportent de la joie et de la compagnie ${ }^{36}$.

Nous voyons là une influence de Westermarck, dont l'œuvre témoigne d'une nostalgie pour la morale des premiers hommes et suggère le malaise de la civilisation moderne. Selon lui, l'histoire de la morale n'a pas toujours marqué un développement vers les idéaux que nous reconnaissons comme les plus nobles. La condition féminine en offre un exemple pertinent. Westermarck, qui devint célèbre pour ses études de l'histoire du mariage, souligne, par exemple, l'effet dégénératif de la civilisation sur la position des femmes et décrit le patriarcat comme un produit de la civilisation. L'idée de la femme comme l'origine du péché et de la mort est née avec le christianisme, suggère Westermarck ${ }^{37}$. Le processus de civilisation a affaibli la position des femmes au lieu de marquer un progrès de l'égalité. Westermarck voit dans le monde des peuples primitifs une égalité innée; une femme peut être égale à un homme, même un dictateur du 
foyer. Chez Lehtonen, la description de la femme et les personnages féminins font également écho aux idées de Westermarck. La tribu de Käkriäinen, de fait, se présente comme une sorte de matriarcat, dans lequel la mère de famille dirige le foyer. Même si Putkinotko a souvent été lu comme une histoire focalisée sur le père de famille, une lecture attentive montre comment la mère, Rosina, joue un rôle central. Elle est représentée non seulement comme le vrai chef de la famille, mais le discours romanesque révèle aussi la portée de sa conscience sociale et de sa réflexion perspicace sur la pauvreté et les structures du pouvoir social. Le père de famille, d'autre part, ne représente pas non plus un stéréotype d'un homme-animal ou d'un homme des cavernes brutal. Même s'il a tendance à avoir des crises de fureur, il résiste à son désir spontané de violence. Ici aussi, le roman de Lehtonen fait écho à Westermarck, qui trouve chez les peuples primitifs une sympathie instinctive envers les autres, et souligne même l'altruisme mutuel des animaux. ${ }^{38}$ La mère de la famille, Rosina, montre de la tendresse envers ses enfants ; elle refuse de les punir en les fouettant comme il en est coutume à l'époque. Le père, Juutas Käkriäinen, s'illustre comme un père aimant, qui nourrit ses enfants en pêchant et en cultivant ses pommes de terre; il gagne son pain quotidien, même s'il a parfois recours à des moyens douteux comme la distillation de la gnôle. «Il aime bien ses enfants et son cochon. Ils sont bien loqueteux, ses enfants. Faudrait leur trouver des vêtements... pourvu qu'on y arrive. ${ }^{39}$ " Les enfants, la joie et l'amour qu'ils apportent à leurs parents et l'espoir qu'ils représentent, se trouvent au cœur même du roman.$^{40}$ Néanmoins, le souci de leur avenir trouble les parents; pendant ses moments les plus mélancoliques, Juutas - qui fait écho à Kullervo, le héros tragique de l'épopée nationale du Kalevala - veut se suicider..$^{41}$ D'une manière tragique, son agressivité se tourne vers lui-même.

Le primitivisme empathique de Lehtonen, qui dirige la sympathie du lecteur vers le peuple primitif, se manifeste aussi dans la description de leur action jugée criminelle : la distillation de gnôle, qui, comme le suggère le sous-titre du roman, fournit un thème central au roman. Angoissée et désespérée par la misère, la famille essaie de trouver un moyen de gagner sa vie et aboutit à distiller illégalement de l'eau-de-vie. L'alcoolisme fut un des problèmes sociaux de l'époque, et au nom de l'abstinence, une loi de prohibition fut appliquée entre 1919 et1932. Toutefois, le roman de Lehtonen refuse une attitude moralisante et culpabilisante envers le peuple et sa consommation d'alcool. La distillerie illégale des pauvres gens est représentée comme une manière de survivre dans les conditions misérables. Le père de la famille, Juutas Käkriäinen, ne boit jamais lui-même et essaie d'obliger ses enfants à s'abstenir de l'alcool. En revanche, ce n'est pas le cas chez les classes supérieures : l'eau-de-vie est vendue à la ville, pour les messieurs et les citadins jusqu'à St. Petersbourg.

19 La description de la visite des Käkriäinen à la petite ville située à proximité fait également allusion aux misères de la vie civilisée ${ }^{42}$. En ville, les aventures de la fille aînée, Saara tournent mal : elle finit, avec un monsieur qu'elle connaît via son travail, par boire de la bière dans un parc et retourne ivre au bateau. La visite de la mère, Rosina, à la pharmacie s'achève par une humiliation profonde : le personnel se moque de cette femme simple, qui ne connaît pas les vrais noms des médicaments et qui demande « un baume de corbeille» pour ses jambes malades. Dans l'officine, elle ne réussit pas à poser une question sur la contraception, à propos de laquelle elle ressent une honte profonde ; la famille Käkriäinen se reproduit si vite que Rosina, anxieuse, essaie de trouver un moyen pour contrôler les naissances. Avec ces scènes critiques, Lehtonen remet l'aspect "civilisé » de la vie urbaine en question : Rosina est bien 
ignorante, mais les « civilisés » se montrent excessivement durs et peu sympathiques à l'égard de cette femme ordinaire.

Toutefois, il convient de remarquer que le ton affectif du roman reste ambivalent. Le sérieux des thèmes sociaux est contrebalancé par les aspects comico-grotesques du texte. Le primitivisme de Lehtonen se manifeste aussi dans le style grotesque qui colore le roman : la vulgarité de sujets "bas» et le vocabulaire vernaculaire qui imprègne l'étude ethnographique caractérisent le texte. Les éléments grotesques se nouent avec l'esthétique de transgression du roman et constituent un contre-pouvoir au contrôle, à l'ordre, à l'harmonie qui définissent non seulement les idéaux de la beauté classique mais souvent aussi notre idée de la civilisation ${ }^{43}$. Par exemple, Putkinotko ne cache pas la corporalité de la vie humaine : la sexualité, la fertilité, voire des sujets scatologiques à la Rabelais sont abordés. La vitalité de l'homme et la nature estivale sont soulignées. La fertilité de Juutas et Rosina établit même un antagonisme entre les classes sociales, car le propriétaire de leur terre n'a pas d'enfants. D'une manière comique, leur crime en commun, la distillation de la gnôle, illustre le pouvoir de la famille et leur capacité à collaborer: la mère, la matriarche, pousse son mari à prendre des mesures; elle persuade son frère de l'aider à cacher les bouteilles d'eau-de-vie aux yeux des autorités. Juutas et ses fils s'occupent de la distillation, et la fille ainée, Saara, en vend car elle travaille comme servante sur un bateau qui assure la liaison jusqu'à St. Pétersbourg en Russie par le canal de Saïmaa.

21 L'accent mis sur la force de la famille, et l'humour et la joie de vivre des enfants en particulier, suggère un espoir dans l'avenir à travers les futures générations. Comme le constate Marianna Torgovnick, une motivation importante pour le primitif dans la littérature moderne fut de créer une nouvelle version de l'idyllique, un primitif utopique, qui renforçait d'anciennes traditions familiales et culturelles, et, en même temps, promettait une ère nouvelle ${ }^{44}$. Dans le roman de Lehtonen, cet aspect idyllique est également renforcé par la peinture de la nature finlandaise, qui évoque les valences positives de la notion du primitif, le vitalisme et l'idéal d'un état authentique. D'une manière métonymique, la description du milieu, à la fois sauvage et belle, reflète des caractéristiques ambivalentes de ses habitants, dont l'extérieur sauvage cache une bonté innée. Le narrateur, le délégué de la civilisation, observe la région primitive et s'extasie même devant le paysage estival :

$\mathrm{Si}$, au milieu de l'été, on gravit ces collines boisées, on peut arriver au sommet d'un monticule d'où la vue, s'ouvrant sur une longue baie, laisse surpris et ébloui. Cette baie peut être d'une telle beauté, d'un bleu si chaud, si brillant et triomphant qu'on a du mal à en croire ses yeux. Merveilleux secrets de ces terres couvertes de lacs !... 45

Le ravissement du narrateur traduit une joie devant le paradis de la nature et exprime une nostalgie pour l'idylle lointaine. Le sentiment de la nostalgie indique une tristesse et un regret provoqués par la distance à l'idylle et par la perte du vieux monde, mais d'un autre côté, il est accompagné d'une consolation: celle de la mémoire d'une belle journée d'été qui restera, même au milieu de l'aliénation de la modernité.

\section{Conclusion}

Dans cet article, nous avons analysé le primitivisme empathique dans le roman Putkinotko de Joel Lehtonen et montré comment une double rhétorique se met en place : 
la perspective anthropologique et civilisée du narrateur est couplée avec le point de vue des pauvres gens et leur expérience de l'oppression. La visée anthropologique du primitivisme se transforme en une esthétique critique qui délivre, et qui, au moyen de l'évocation des émotions, est destinée à stimuler le sens éthique du lecteur, une empathie réflective qui met en question les clichés du primitivisme colonisant. Cet esprit de la transgression, qui se développe dans l'écriture de Lehtonen, tient à contester les hiérarchies et les oppositions entre la civilisation et le monde primitif. En fin de compte, le ton affectif du roman reste ambivalent. La description grotesque et naturaliste des personnages, qui suscite des moments de rire carnavalesque, se fond avec la mélancolie du paradis perdu. En dépeignant une idylle qui se brise, Lehtonen élabore une image nostalgique du vieux monde prémoderne et primitif qui fait face aux défis du monde moderne tout en restant connecté aux problèmes sociaux du monde rural de l'époque.

\section{NOTES}

1. Joel Lehtonen, La Combe aux mauvaises herbes, traduit du finnois par Jaakko Ahokas, collection U.N.E.S.C.O d'œuvres représentatives, Paris, Del Duca, 1962, p. 13-15. Joel Lehtonen, Putkinotko, Helsinki, SKS, 1995. Le texte original en finnois : «Savon eteläosassa, lähellä Karjalan heimon asuinseutujen rajaa, on karu ja kaunis paikkakunta. Saimaa, joskus muualla leveä ja vapaa, supistuu siellä ahtaiden maisemien väliin, niin että ainoastaan harvoin saattaa nähdä ulapalla sellaisen kohdan, jossa silmä ei tapaa missään toista rantaa: vedenkalvo yhtyy siintävään avaruuteen kuin merellä. [--] Tämä sisämaa on melkein yhtä karua kuin rannikko. Taloja on harvassa, ja niitäkin erottavat toisistaan ainaiset vesien taikka mäkien solat, lahdet ja puolittain umpeen sammaltuneet lammet. [--] Maan karuudesta ja vesien esteistä johtuneekin osaltaan, että tällaisella seudulla saattaa tavata kansaa, joka on, paitsi yleensä köyhää, joskus siinä määrin kehittymätöntä, saamatonta, itsepintaisen kulmikasta ja taikauskoistakin, että mieleen johtuu melkeinpä kaikkein alkeellisin metsäläisyys. Ja tämä seikka on sitä merkillisempi, kun sivistyskeskusta, kaupunki, on ainoastaan peninkulman päässä. » Voir Lehtonen 1995, p. 3-5.

2. Jaakko Ahokas a traduit le sous-titre du roman par «l'Histoire du Philanthrope naif et du Bouilleur de Cru paresseux. " La traduction n'est pas littérale, ni très objective si on prend en considération l'antagonisme des classes sociales décrit par le roman. « Tuhma » ou » tyhmä » ne signifie pas seulement « bête » en français, mais se traduit aussi par « méchant ». Dans cet article, nous citons la traduction d'Ahokas, avec quelques petites modifications et corrections du texte en français.

3. Voir Magnus Björkenheim, Joel Lehtosen Putkinotko, 1955, Helsinki, Suomalaisen Kirjallisuuden Seura, p. 44.

4. Edvard Westermarck, Ur sedernas historia. Föredrag hållna i Åbo hösten 1911. Helsingfors, Söderström \& C/o förlagsaktiebolag, 1912; Edvard Westermarck, Tapojen historiaa. Kuusi akadeemista esitelmää pitänyt Turussa syksyllä 1911, traduit du suédois par Joel Lehtonen, Helsinki, Suomalaisen Kirjallisuuden Seura, 1991.

5. Joel Lehtonen, Puolikuun alla. Matka- ja mielikuvia murjaanien maasta, Hämeenlinna, Arvi A. Karisto, 1919. 
6. Riikka Rossi, Alkukantaisuus ja tunteet : primitivismi 1900-luvun alun suomalaisessa kirjallisuudessa, Helsinki, Suomalaisen Kirjallisuuden Seura, 2020.

7. Kai Laitinen, Littérature de Finlande en bref, traduit du finnois par Jean-Michel Kalmbach, Helsinki, Otava, 1997, p. 87.

8. La Finlande est devenue indépendante en 1917.

9. Voir aussi Pirjo Lyytikäinen, «Decadence in the Wilderness: Will to Transgression or the Strange Bird of Finnish Decadence » in Nordlit 28, (2): p. 245-56. https://doi.org/10.7557/13.2062. Consulté le 30.09.2020.

10. Ben Etherington, Literary Primitivism, California, Standford University Press 2017, p. xii-xiii.

11. Ben Etherington, Literary Primitivism, p. 9.

12. Sur cette double rhétorique, voir Riikka Rossi, Alkukantaisuus ja tunteet, p. 28.

13. Ben Etherington utilise la notion du "primitivisme emphatique " largement pour renvoyer à la manière dont une œuvre primitiviste tient à atteindre l'état considéré comme primitif. Ici, nous reprenons ce terme pour illustrer les effets émotionnels et les techniques narratives du primitivisme critique. Voir Ben Etherington, Literary Primitivism p. 10 et 45 et Riikka Rossi, Alkukantaisuus ja tunteet, p. 25.

14. Né hors mariage, fils d'une servante, Joel Lehtonen est d'origine pauvre. Il fut abandonné par sa mère à l'âge de six mois, et, comme c'était la coutume à l'époque, fut «vendu » par la paroisse à qui promettait de s'occuper de l'enfant au plus bas prix. Il devint l'enfant adoptif d'une veuve de pasteur, qui lui ouvra la voie des études jusqu'à l'université de Helsinki. Sur la vie de Lehtonen, voir Pekka Tarkka, Joel Lehtonen 1. Vuodet 1881-1917, Helsinki, Otava 2009, et Joel Lehtonen 2, Vuodet 1918-1934, Helsinki, Otava 2012.

15. Voir Michael Bell, 2010, «Primitivism. Modernism as anthropology " in Brooker, Peter, GĄsiorek, Andrzej, Longworth, Deborah \& Thacker, Andrew (éd.) The Oxford Handbook of Modernisms, Oxford, Oxford University Press, p. 354.

16. Arthur Lovejoy \& George Boas, Primitivism and Related Ideas in Antiquity, Johns Hopkins University Press, 1997 (1935).

17. Marianna Torgovnick, Gone Primitive: Savage Intellects, Modern Lives, Chicago \& London: The University of Chicago Press, 1990; Marianna Torgovnick, Primitive Passions: Men, Women and the Quest for Ecstacy. Chicago \& London : The University of Chicago Press, 1996.

18. Ce chiasme entre le moderne et le primitif s'étend aussi à l'antagonisme entre la cité et la forêt : comme l'a noté Robert Harrison, l'Occident a défriché son espace au cœur des forêts et fondé contre elles ses institutions dominantes, comme la cité. Mais, en même temps, la lisière ténébreuse des forêts ne cesse d'interroger l'imaginaire occidental. Voir Robert Harrison, Forests: The Shadow of Civilization, Chicago, University of Chicago Press, 1992, p. 6.

19. Voir Bell, p. 353-367. De même, comme le constate Dominique Viart dans son analyse sur le primitivisme de William Faulkner, la recherche de la mentalité primitive du premier homme " authentique » a servi à développer des nouvelles techniques comme le fleuve de la conscience ou le monologue intérieur. Voir Dominique Viart, «Un mausolée pour le Sud. Faulkner et les paradoxes du primitivisme », in Bernard Mouralis (éd.) Primitivismes. Revue des Sciences humaines, $\mathrm{n}^{\circ} 227$, p. 197-229. Voir, surtout, p. 209-210.

20. Voir Joel Lehtonen, Putkinotko, p. 33 et Joel Lehtonen, La Combe aux mauvaises herbes, p. 40.

21. C'est surtout le poème «Bonden Pavo» («Le paysan Paavo»), par J. L. Runeberg, du recueil Dikter (Poèmes, 1830), qui établit le modèle de l'homme finlandais convenable pour l'idéologie du mouvement nationaliste.

22. Voir Sara Ahmed, The Cultural Politics of Emotion, Second Edition, Edinburgh : Edinburgh University Press, 2014, p. 131.

23. Sur la pauvreté rurale dans la Finlande du XIX ${ }^{\mathrm{e}}$ siècle, voir par exemple Miikka Voutilainen, Poverty, Inequality and the Finnish 1860s. Jyväskylä : University of Jyväskylä, 2016, http://urn.fi/ URN:ISBN:978-951-39-6627-0 Consulté le 30.09.2020. 
24. Il est à souligner que dans le cas de Lehtonen, la notion du colonialisme se réfère à une analogie que propose le récit fictif, plutôt qu'au sens historique du terme, c'est-à-dire à l'exploitation des territoires en dehors des frontières nationales. De fait, les récits du primitivisme décrivent le colonialisme non seulement en tant qu'un phénomène historique de la politique impérialiste, mais mettent également en lumière les attitudes coloniales en général. Comme l'affirme Ben Etherington, le primitivisme littéraire a surgi largement contre les structures du pouvoir et de la domination sociale de l'époque capitaliste. Ibid. p. 8.

25. Sur la notion de l'empathie narrative, voir Suzanne Keen, Empathy and the Novel, Oxford: Oxford University Press, 2007, p. 5.

26. Joel Lehtonen, La Combe aux mauvaises herbes, p. 49. Pour le texte en finnois, voir Joel Lehtonen, Putkinotko, p. 42 : «Mutta samalla sekaantuu siihen pellon mieluiseen ajatteluun katkeruus ja kiukku, ettei se pelto, joka on heidän, Putkinotkon mökin asukkaiden, raivaama ja reuhtoma, ole oikein pohjiaan myöten hänen eikä heidän. Se katkeruus on kurkussa kuin suola ». 27. On peut mentionner, par exemple la célèbre figure de Homsantuu dans Työmiehen vaimo (1885, La femme d'un ouvrier) par Minna Canth, qui, à la fin du drame, s'oppose à la police et au pouvoir patriarcal.

28. Joel Lehtonen, La Combe aux mauvaises herbes, p. 320, et Joel Lehtonen, Putkinotko, p. 319 : «Kipeästi koskee Juutaksen rintaan. Suunnattoman kipeästi se koskee. Putkinotkossa on hän ollut toistakymmentä vuotta, ja tuuminut, miten hän siellä suonkin perkkaisi. Nyt vievät muut kaikki, mitä hän on sinne jo tehnyt. Ja ne tekevät sinne omia laitoksiaan. Repiä ne pitäisi kaikki pois. Potkia tieltä ...»

29. Riikka Rossi, Alkukantaisuus ja tunteet, p. 53.

30. Voir Joel Lehtonen, La Combe aux mauvaises herbes, p. 496, et Joel Lehtonen, Putkinotko, p. 499 : « Kuinka syvän ja intohimoisen vihan näin Rosinan silmissä kiehuvan silloin, kun pakotin hänen miehensä pykäliini ! Ja muistatko, mitä Rosina sanoi varsinkin viime kesänä ? Jos huomasi meidät mökin pihalla tai pientarella, purki hän sydäntään, ikäänkuin ainoastaan toisille Käkriäisille, mutta tarkoitti sanansa minulle. Näin hän sanoi : Miksi täällä kyntämään ja kylvämään, toisen tilalla, josta voidaan ajaa pois?»

31. « Metsän villien asukkaiden loppu on aina tavallaan traagillinen » Joel Lehtonen, Putkinotko, p. 515.

32. Voir, par exemple, Lucien Lévy-Bruhl, La Mentalité primitive, Paris, Librairie Félix Alcan, 1922.

33. Selon William Reddy, les émotions constituent une sorte de précondition pour la volonté et la motivation. Voir William Reddy, The Navigation of Feeling: A Framework for the History of Emotions. Cambridge, U.K. : Cambridge University, 2001, p. 120.

34. L'émotion de sisu, qui est difficile à traduire dans les langues étrangères, a été tenue pour une caractéristique importante de la mentalité finlandaise. Dans l'histoire, sisu a été interprété de plusieurs façons, de l'idée d'un personnage bilieux, hargneux et rigide jusqu'à l'expression de la persévérance et du courage. Voir, par exemple, Tuomas Tepora, «Sisu: The Finnish for Stiff Upper Lip. », 30 octobre 2012, https://emotionsblog.history.qmul.ac.uk/2012/10/sisu-the-finnish-forstiff-upper-lip/ Consulté le 30.09.2020.

35. Dans le cadre de la tradition romanesque finlandaise, la passion des Käkriäinen fait aussi écho au célèbre roman Seitsemän veljestä (tr. en français Les Sept frères d'Aleksis Kivi), dans lesquels les frères sont décrits comme enclins à devenir coléreux et tomber dans la passion. De même, la mise en exergue de leur colère, fait penser au stéréotype de l'homme finlandais créé par Zacharias Topelius, Matti, qu'il décrit dans son célèbre manuel scolaire, Boken Om Vårt Land (Le livre sur notre pays, 1875) : Matti est laborieux et aime sa patrie, mais quand il se met en colère, sa colère n'a aucune limite.

36. Après la parution de Putkinotko, un critique finlandais a comparé l'œuvre à La Terre de Zola, un roman qui, parmi les œuvres souvent urbaines de Zola, représente une étude sur le monde rural. Certainement, la peinture nue des «sauvages» finlandais chez Lehtonen a été influencée 
par l'écriture et l'esthétique zolienne. Mais si chez Zola les paysans sont représentés comme des êtres sans émotions, indifférents à l'égard de leurs prochains et capables de transgresser les tabous humains (comme en témoignent les thèmes du parricide et du viol dans le roman), Lehtonen garde pourtant une nostalgie pour un « sauvage noble » qui, malgré les apparences peu convenables, a un bon cœur. Chez Zola, le monde rural est souvent peint comme la plus basse classe sociale, inférieure même à celle des ouvriers.

37. Voir Edward Westermarck, Ur sedernas historia, p. 26.

38. Westermarck, Ur sedernas historia, p. 61-64.

39. Voir Lehtonen 1962, p. 134 ; Lehtonen 1995, p. 131 : «Rakastaapa hän lapsiaan ja omaa sikaansa. Repalaiset nuo ovat lapset. Pitäisi niille hankkia vaatteita ... kun tulisi hankituksi. »

40. Sur le rôle des enfants, voir Aarne Kinnunen, Joel Lehtosen Putkinotko, Helsinki, WSOY 2005, p. 290.

41. Pekka Tarkka, Putkinotkon tausta. Joel Lehtosen henkilöt 1901-1923, Helsinki : Otava p. 296-298.

42. Dans Putkinotko, Lehtonen crée un univers romanesque imaginaire qui prend une certaine distance avec les réalités historiques. Le nom de Savonlinna, par exemple, n'est jamais mentionné dans le roman. Pourtant, la ville imaginaire décrite dans Putkinotko a une nette ressemblance avec la ville réelle de Savonlinna, avec la forteresse, l'établissement de bains et la communication fluviale sur le lac Saïmaa ; la famille Käkriäinen voyage de sa métairie à Savonlinna par le bateau, comme cela se faisait à l'époque.

43. Comme l'a décrit l'historien d'art Ernst Gombrich, dans l'art occidental il existe une sorte de préférence pour le primitif qui fournit un contre-courant aux idéaux classiques de la beauté et de l'harmonie. Ernst Gombrich, The Preference for the Primitive. Episodes in the History of Western Taste and Art, London, Phaidon Press, 2002.

44. Marianna Torgovnick, Gone Primitive,1990, p. 245.

45. Joel Lehtonen, La Combe aux mauvaises herbes, p. 16-17, et Joel Lehtonen, Putkinotko, p. 6-7 : «Jos sydänkesällä kiipeilee siellä metsäisillä kukkuloilla, niin voi joutua jollekin kummulle, mistä näkee pitkälle lahdelle, ja silloin hämmästyy ja huikaistuu. Niin kaunis saattaa se lahti olla, niin lämpöisen sininen väriltään, ja loistava ja riemukas, ettei melkein usko silmiään! Järviseutujen ihanuutta !...»

\section{RÉSUMÉS}

Dans cet article, nous nous proposons d'étudier, à travers le roman Putkinotko (1919-1920) de Joel Lehtonen, les formes du primitivisme finlandais et d'examiner les rencontres du primitif et du civilisé dans l'imaginaire de l'époque. Dans la littérature finlandaise de l'époque, les classes agraires sont devenues essentielles pour imaginer le primitif entre nous et pour raconter l'histoire d'une jeune nation à l'aube de la modernité. En outre, l'analyse du roman de Lehtonen nous permet de réfléchir sur l'idée du primitivisme en tant que conception esthétique et courant littéraire. Même si le primitivisme a été fréquemment considéré comme un projet colonisant de l'époque impérialiste, il nous semble nécessaire de nuancer et de réviser ce point de vue afin de mettre l'accent sur le potentiel critique du primitivisme littéraire et artistique, voire sur son horizon décolonisant. Dans le roman de Lehtonen, une double rhétorique se met en place: le regard et la voix scientifique et civilisée du narrateur sont couplés avec le point de vue des pauvres gens et leur expérience de l'oppression. La visée anthropologique se transforme en une esthétique critique au moyen de l'évocation des émotions, et est destinée à stimuler le sens 
éthique du lecteur, une empathie réflective qui met en question les injustices sociales décrites dans le roman. Cet esprit de la transgression, qui se développe dans l'écriture de Lehtonen, tient à contester les hiérarchies et les oppositions entre la civilisation et le monde primitif.

This article explores forms of primitivism in the novel Putkinotko (1919-1920) by Joel Lehtonen and analyses the encounters of the primitive and the civilized in the imagination of the era. In early nineteenth-century Finnish literature the agrarian classes became essential to imagining the "primitive in us" and to narrating a nation on the edge of modernity. Rural people were compared to exotic tribes or seen as archaic remnants in a civilization where the primitive was still observable in its full vitality. However, the analysis of Lehtonen's novel permits us to rethink the idea of primitivism as an aesthetic concept and as a literary current. Particular attention is paid to the critical and de-colonial potential of primitivism: the Finnish primitivism analysed here in many respects represents "emphatic primitivism", which discovers discontentment with civilization from the perspective of the primitive and "feels with" the primitive. The novel of Lehtonen is characterized by a double rhetoric: the anthropological gaze of the narrator is coupled with the point of view of the primitive people and their experience of oppression. While primitivism is an ambivalent concept that has been employed to legitimize various ideological and political views, the narratives of the primitive frequently express a critical stance towards the civilization they escape and challenge the hierarchies and attitudes of the social domination they depict.

\section{INDEX}

Mots-clés : littérature finlandaise, primitivisme, Lehtonen (Joel)

Keywords : Finnish literature, primitivism, Lehtonen (Joel)

\section{AUTEUR}

\section{RIIKKA ROSSI}

Riikka Rossi est maître de conférences en littérature finlandaise à l'université de Tampere et à l'université d'Helsinki en Finlande. Elle est spécialiste du naturalisme, de la littérature du $\mathrm{XIX}^{\mathrm{e}}$ siècle et de la théorie sur la littérature et les émotions. Riikka Rossi est également éditrice de nombreux volumes d'articles et auteure, entre autres, d'un ouvrage sur le primitivisme finlandais (en finnois, Alkukantaisuus ja tunteet, SKS 2020). Elle dirige actuellement un projet de recherche sur les émotions dans les récits du nord, intitulé L'Hysterie arctique - les étranges émotions nordiques (2020-2022) financé par la fondation Kone (Finlande). 